\title{
Layered Dynamic Control for Interactive Character Swimming
}

\author{
Po-Feng Yang, Joe Laszlo and Karan Singh ${ }^{\dagger}$ \\ Department of Computer Science, University of Toronto
}

\begin{abstract}
This paper proposes a layered strategy for controlling character motion in a dynamically varying environment. We illustrate this approach in the context of a physically simulated human swimmer. The swimmer attempts to follow a dynamic target by augmenting cyclic stroke control with a set of pre-specified variations, based on the current state of the character and its environment. Control of a given swim stroke is decomposed into three layers: a basic stroke sequence, a set of per-stroke control variations, and a set of continuously applied control variations. Interactive control of the swimmer is possible as a result of an efficient physical simulation using a simplified fluid model. Our results show layered dynamic control to be an effective adaptive control technique in well conditioned physical simulations such as swimming, where simulation states resulting from control errors are recoverable.
\end{abstract}

Categories and Subject Descriptors (according to ACM CCS): I.3.3 [Computer Graphics]: Interaction, Physical simulation, Fluids, Character animation

\section{Introduction}

Computer animation research has produced a number of models for synthesizing physically simulated motion of virtual humans. Much of this research has addressed the control of specific forms of motion such as walking [LvF96], running, cycling [HWB*95], and acrobatics [WH96]. Character motion in fluids is a relatively unexplored area, with prior work primarily addressing locomotion of non-human characters such as evolving creatures [SIM94] and fish [TT94] in water or birds in air [WP03]. Character state during swimming is relatively stable and changes smoothly compared to motions such as walking that take place on the fringes of stability. Swimming thus has a graceful unique visual quality unlike other motions to an extent that the term "fluid motion" is often used to describe the visual appearance of any graceful movement. Inspired by the desire to generate "fluid character motion" at a rate suitable for interactive control, this paper addresses the control and animation of swimming human figures as shown in Figure 1.

Our approach is based on the physical simulation of a

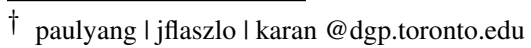

dynamic human figure model in a simplified fluid model. The simulated swimmer follows a target under interactive user control. Basic swim strokes are defined using a cyclic pose control graph (PCG) driving joint-level proportionalderivative (PD) controllers to generate coarse overall motion for the stroke. The smooth transitions of character state when swimming, relative to say, walking, allows us to apply layered control variations to the base control of a stroke in order to realize goal directed behaviour, such as target following, under a variety of environmental conditions. The decomposition of our swimmer's motor control system into three independent layers, shown in Figure 2, makes the overall control system significantly less complex than might otherwise be required for comparable results.

In addition to the basic stroke control layer, a per-cycle control layer applies stroke variations on a per cycle basis to effect desired changes primarily in the character's global state, such as for directional control. These variations are defined heuristically, based on techniques developed by swimming coaches, observing real swimmers and direct experimentation with the virtual swimmer. A continuous perturbation layer continuously adapts the character pose locally to the current state of the environment, such as adjusting the 

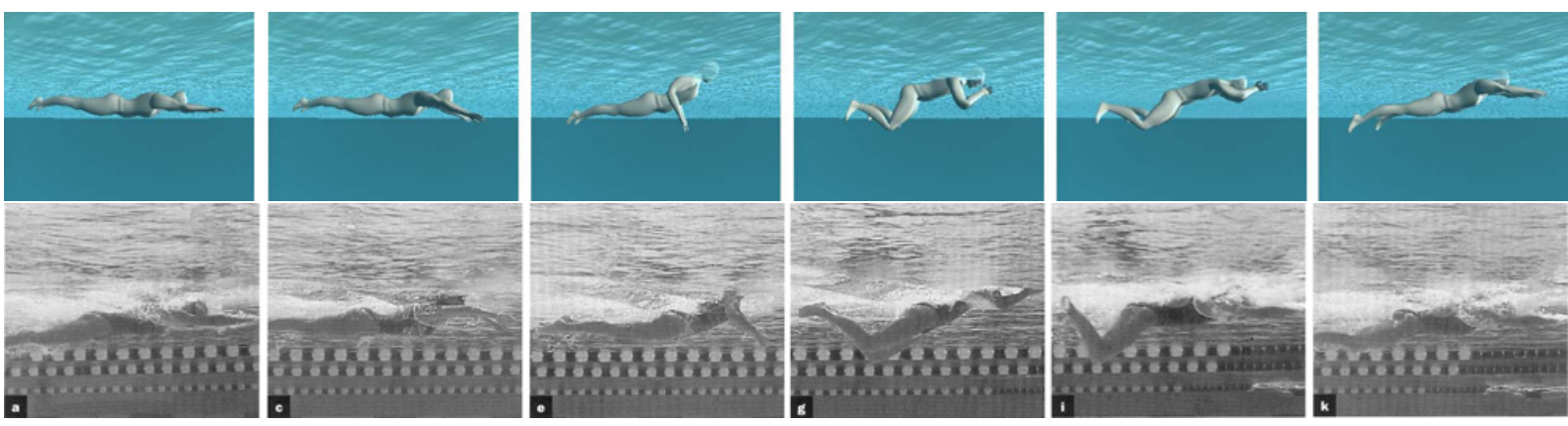

Figure 1: Comparison between a synthesized breast stroke and a real swim sequence (from [MAG03]).

wrists based on the local relative fluid velocity to increase thrust or decrease drag. At a higher level the selected stroke with which to follow a target is typically specified by the animator either interactively or through scripting.

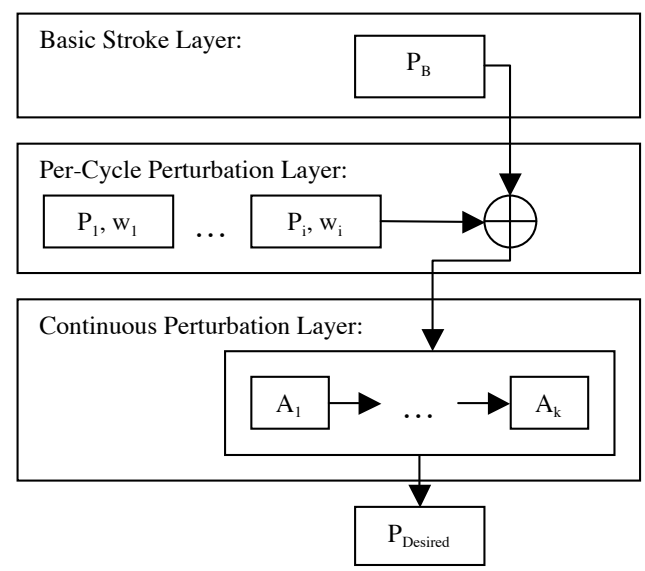

Figure 2: Swimmer architecture: The basic stroke layer cycles through a PCG to generate a pose $P_{B}$. The per-cycle perturbation layer blends a set of pose perturbations $P_{i}$, with $P_{B}$. The continuous perturbation layer makes local pose changes, adapting blended pose to the dynamic environment.

This paper thus contributes a system to generate visually realistic swimming characters under interactive control. The animation algorithm is based on independent layers of dynamic control, which is shown to be an effective strategy for simulated characters in well conditioned environments (where errors in control input are not irreversible). The generated swimming motion finds applications in animation, games, for swimmer education or as an analysis tool for exploring different swimming strokes.

The rest of the paper is organized as follows: Section 2 reviews related work. Section 3 describes the swimmer, fluid and their interaction model used to perform the physical simulation. Section 4 describes our layered dynamic control al- gorithm. Section 5 provides implementation details and discusses the resulting animations created by our system. Section 6 concludes with directions for future research.

\section{Related work}

Our work is related to four areas of research: dynamic control for humanoids, dynamic control in a fluid environment, fluid solid interaction, and the biomechanics of swimming.

\section{Controlling Human Motion}

Algorithms for physics-based running, cycling, vaulting and platform diving were described in [HWB ${ }^{*}$ 95] and [WH96]. The character is modelled using a system of rigid bodies and muscle force using PD controllers. Biped walking has also received a lot of attention in the research community. [LvF96] described a dynamic control algorithm for physically based walking animation based on the idea of limitcycles. [FvT01] showed the benefits of solving complex dynamic control problems by sequentially applying various simple dynamic controllers. This paper illustrates that composing various simple controllers in a layered fashion in well conditioned simulation environments can also provide an effective solution to complex dynamic control problems. Our system, like the body of research above, is based on physical simulation, without which achieving visual realism of complex figures in a dynamic environment is a difficult problem.

\section{Dynamic Control in a Fluid Environment}

In prior research on controlling human motion, the physical environment does not influence a character in the immersive way in which a fluid field does. The interaction between a swimmer and fluid has been addressed by [vF93] and [TT94] for locomotor control of fish in 2D and 3D respectively. In [vF93], a simple 2D "fish" character learns robust target following through stochastic optimization of controller parameters. In [TT94] manually-designed locomotion controllers provide the foundation for rich behavioural repertoires of fishes living and interacting in a virtual marine world. Modeled as spring-mass systems with a simple sinusoidal actuator propelling the fish through water, low-level controllers were directed by a behavioral model to make 
the fish swim, turn and perform higher-level functions such as schooling. Such a behavioral system could be used to specify the target followed by our virtual swimmer. [GT95] automatically synthesized similar low-level undulatory motor control by stochastic optimization in the frequency domain. In [SIM94], complex swimming figures learned to swim by evolving both skeletal structure and control parameters using genetic algorithms. Research on flight [WH91] [WP03] looks at the effects of aerodynamic forces. [WH91] linearized the Navier-Stokes equation to provide a set of primitives to define flow fields for animating aerodynamics. [WP03] parameterized bird flight using a small set of wing beat parameters to control complex physical models and proposed a generalized optimization algorithm to solve for optimal parameters for different bird models performing various maneuvers by attempting to satisfy a user-defined trajectory.

\section{Fluid Solid Interaction}

The hydrodynamic or aerodynamic models used in the previous section are highly simplified. More sophisticated models for animating fluid solid interaction is an active area of research [GHD03] [CMT04]. [GHD03] uses a spring mass system to model the interaction between deformable objects and fluid. [CMT04] solves the problem of rigid body and fluid interaction by incorporating the rigid body constraints into the boundary condition in the fluid.

\section{Biomechanics of Swimming}

A wealth of research can also be found on human swimming in the biomechanics and athletic training community. Research on swimming biomechanics is primarily focused on analyzing drag and lift acting on a swimmer's body, a survey of which can be found in [MAG03]. [BdH*95] and $\left[\mathrm{PKH}^{*} 00\right]$ use mechanical models to measure the drag and lift on hands, arms, legs and feet of a swimmer while [BR02] uses CFD (computational fluid dynamics) to simulate the flow around a static arm mesh. [SH03] analyzes the forces on an arm model during motion captured from a real swimmer. We use the observations in [SH03] to motivate the segmentation of swimmer control into the three independent layers seen in Section 4.

\section{Swimming Dynamics Simulation}

Our system simulates the dynamics of swimming using rigid bodies and a simple fluid mechanics model.

\subsection{Swimmer Model}

The character is modeled by 16 segments of rectangular rigid bodies connected by 16 joints and 39 degrees of freedom (DOF) to form a humanoid swimmer. A mixture of Euler angle and quaternion (ball joint) representations are used. Figure 3 shows the DOF of our virtual character. Parameters for the rigid bodies are obtained from [WH96]. The total weight of the swimmer is $89.57 \mathrm{~kg}$ and the density is $1.044 \mathrm{~g} / \mathrm{mL}$ making the swimmer sink in water. Breathing has a significant impact on the torso volume and thus on the buoyancy of the swimmer. The parameters in [WH96] are based on cadaveric data, where the measured volume of the torso is lower than that of a live swimmer with air filled lungs. We increase the torso volume by up to $3 L$ empirically, which results in a buoyancy of $91 \mathrm{~kg}$ in water, transitioning our swimmer from a sinker to a floater. The muscles of the virtual swimmer are modeled by PD controllers.

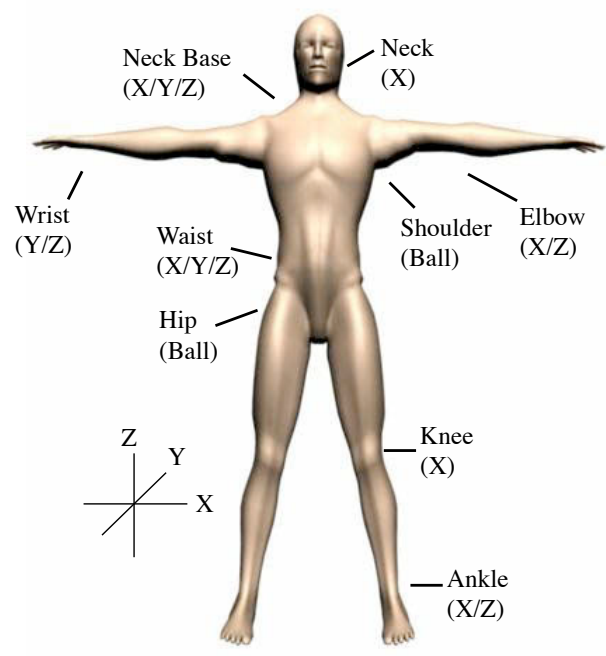

Figure 3: Joint DOF in the swimmer model. The character is initialized to lie on its stomach in the pose shown.

\subsection{Fluid Model}

We use a simple fluid model in our simulation. The hydrodynamic force is based on a thin plate model from fluid dynamics literature [HOE65][HB75]. Buoyancy is approximated using bounding ellipsoids. The flow field does not dynamically interact with the character.

\section{Hydrodynamic forces}

We separate the hydrodynamic forces into drag, lift and friction. Drag and lift are generated by displacing fluid molecules while the swimmer moves its body. Friction comes from the attraction between the body surface and the fluid molecules due to viscosity. Assuming the fluid has steady flow as does most biomechanics literature on swimming [TOU02], the magnitude of the drag and lift can be computed as:

$$
f=\frac{1}{2} C(\alpha) \cdot \rho \cdot a_{n} \cdot\left|v_{n}\right|^{2}
$$

where $C(\alpha)$ is the drag/lift coefficient, $\rho$ is the density of the fluid, $\mathrm{a}_{n}$ is the projected frontal area of the polygon, and $v_{n}$ is the normal component of the relative velocity of the fluid. The magnitude of the friction force is computed by 


$$
f=\eta \cdot a_{t} \cdot v_{t}
$$

where $\eta$ is fluid viscosity, $a_{t}$ is the projected tangential area and $v_{t}$ is the tangential component of the viscosity. The forces are applied along vector $D, L$ and $T$ as shown in Figure 4. Given the normal of the polygon, $N$, and the relative velocity of the fluid, $V$, we consider the hydrodynamic model to determine $D, L, T$ and $C(\alpha)$. The model we use is a thin rectangular plate with aspect ratio 0.2. We use an extra forward vector, $F$, to indicate the long side of the rectangle. $D$ is the normalized projection of $V$ on the plane spanned by $N$ and $F . L$ and $T$ are defined as follows:

$$
U=\frac{D \times F}{|D \times F|}, L=\frac{D \times U}{|D \times U|}, T=\frac{V-(V \cdot D) D}{|V-(V \cdot D) D|}
$$

The angle of attack, $\alpha$, is computed as:

$$
\alpha=\cos ^{-1}(D \cdot N)
$$

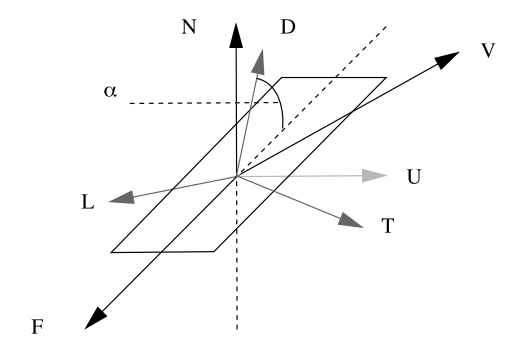

Figure 4: Applied fluid force direction vectors.

The drag and lift coefficient can be approximated by interpolating the results summarized in Figure 5. Only front facing polygons $(N \odot V>0)$ contribute to force. Back facing polygons are assigned a force of magnitude zero.

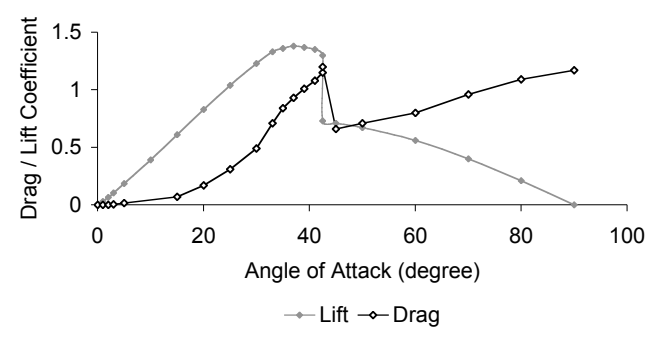

Figure 5: The drag and lift coefficient of a fully immersed thin plate at a given angle of attack for a low Reynolds number. The data is obtained from [HOE65] and [HB75].
We sum up forces on all polygons of a segment and apply the external force and torque coming from the fluid to the corresponding rigid body.

\section{Buoyancy}

We approximate the effect of buoyancy based on the submerged ratio the ellipsoids representing each body part, computed as illustrated by Figure 6. Since the ratio of volume is preserved under affine transformation we compute this ratio by first finding the point of intersection $I_{E}$ of the fluid surface with a principal axis of the ellipsoid. This point is transformed back to a point in the unit sphere $I_{C}$ subtending an angle $\theta$ as shown in Figure 6. The ratio of submerged ellipsoid volume is then $(1+\cos (\theta)) / 2$.

\section{Swimmer-fluid interaction}

The motion of the swimmer through the fluid flow field does not influence the fluid flow field in our simplified fluid model. We approximate the flow of the fluid around the character based on the swimmer's velocity and orientation. $V_{F}$, the velocity of the fluid near the swimmer, is set to be proportional to $V_{S}$, the swimmer's velocity. $V_{F}$ is determined. We use $\sin ^{3}$, the drop-off function of a cylinder's drag coefficient in [HOE65] to approximate the effect of profile drag at different angle of attack, specifically:

$$
V_{F}=\left(\left(r_{\text {Max }}-r_{\text {Min }}\right) \sin ^{3}(\alpha)+r_{\text {Min }}\right) V_{S}
$$

where $r_{M a x}$ and $r_{M i n}$ are user defined maximum and minimum ratios and $\alpha$ is the angle between $V_{S}$ and the $z$-axis of the swimmer's pelvic frame. In addition, the user can create any desired flow field using primitives defined in [WH91].
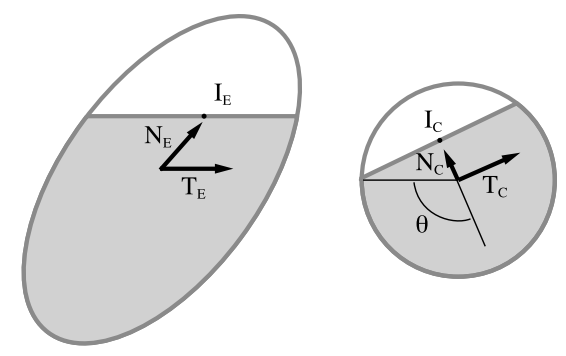

Figure 6: The ratio of volume of partially submerged ellipsoid is computed by transforming it to a unit sphere.

\section{Dynamic Control}

Given the physical models of the swimmer, fluid and their interaction, we now describe the layered dynamic control algorithm that drives the swimmer to follow an interactively specified 3D target. There are three layers in our system as shown in Figure 2. Each layer operates independently and the combined results determine the desired swimmer pose 
used to drive joint PD-control set-points. Figure 8 summarizes the different effects of each layer on the resulting motion.

\subsection{Well-conditioned environment}

The layered strategy for interactive dynamic control is based on an implicit assumption that the forces in our fluid environment are continuous and that there are no character states in the system from which the controller cannot recover. This allows us to separate global control of the character into independent layers that are applied greedily over a current window of time, instead of using global optimization given a pre-specified trajectory as in [WP03]. While general fluid environments can be quite turbulent, our simplified steady flow model produces smooth interaction forces between the fluid and the swimmer. As an example Figure 7 shows the external forces on the swimmer's left hand during one breast stroke cycle. Such a control strategy is more difficult to apply to motions such as walking, where contact forces result in sharp discontinuities in the profile of forces over time and controllability is intermittent. This paper exploits this wellconditioned property of a fluid environment and proposes a continuously adapting scheme in the continuous perturbation layer to augment the stroke.

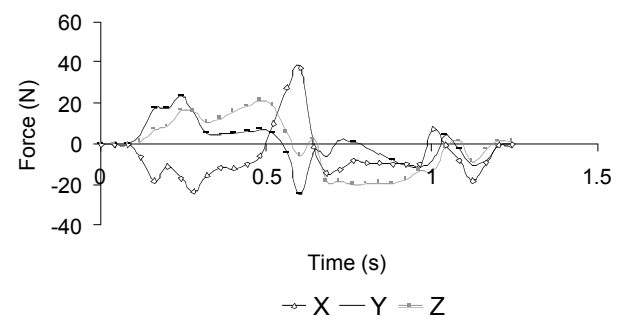

Figure 7: External force on the left hand of the swimmer expressed in world space. One cycle of the breast stroke with the character starting at rest is shown.

\subsection{Basic Stroke Layer}

The basic stroke layer takes a sequence of poses defining a stroke cycle and interpolates the appropriate pose for the swimmer at the current time. Figure 9 illustrates the manner in which the desired pose drives the motion of the character. This layer determines the basic form of a stroke used by the swimmer. The pose cycles for strokes in our system are defined empirically based on their verbal and visual description in [MAG03]. Key poses are defined for each joint separately, reducing redundant poses in our sequence. In the breast stroke, for example, the legs are not actively moving during the sweeping phase of the arms and need not to be explicitly specified. Independently keying joints also makes the incorporation of pose perturbations from other layers straightforward.

\subsection{Per-Cycle Perturbation Layer}

This layer defines perturbations to the basic stroke cycle as motion modifiers to empirically accomplish specific tasks like turning while swimming. These perturbations, like the basic stroke layer, are empirically defined. In our system we use this layer to change the direction of the swimmer using six perturbation sequences. These sequences correspond to the modifications to a basic stroke that the swimmer would employ to achieve pitch, yaw and roll in either direction. We define these perturbations relative to the basic stroke.

The input to this layer is the current simulation time, target position, and the interpolated pose from basic stroke layer. At the beginning of each stoke cycle, a weight is determined for each perturbation sequence and this weight scales the values of perturbation to be applied for that cycle. The weights are computed based on the position and orientation of the swimmer relative to the target. Through preprocessed simulations of the swimmer at various velocities in a static fluid, we determine the weight value needed to turn the swimmer through a given angle. The weights scale and blend the six perturbation sequences with pose sequence output from the basic stroke layer. Figure 10 illustrates one cycle of turning left while treading water using this layer. Figure 11 shows the change in character orientation over time.

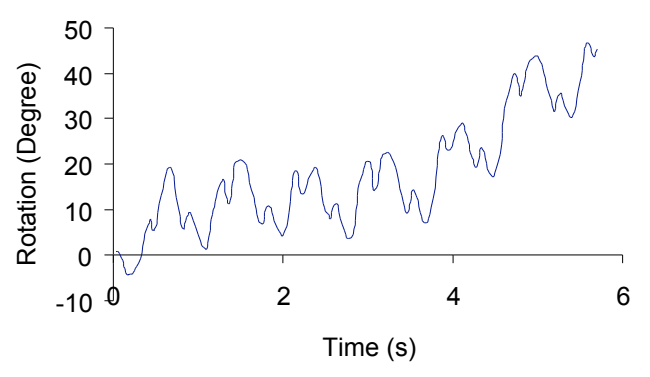

Figure 11: Turning left while treading water. Rotation is measured about the character's yaw $(Y)$ axis.

\subsection{Continuous Perturbation Layer}

The continuous perturbation layer allows us to dynamically vary the motion of a character in a local fashion based on the current environment and a set of heuristics that are generally valid in well-conditioned environments. The input to this layer is the target position, the blended pose from the per-cycle perturbation layer and a set of rules. These rules are based on the intuitive strategies people take when swimming and are applied to modify the blended pose continuously. Currently, we apply two rules in our system seen in Figure 12:

- Palm Orientation The palms should face into the direction of the fluid if more force in required in the desired 
P. Yang, J. Laszlo \& K. Singh / Layered Dynamic Control for Interactive Character Swimming
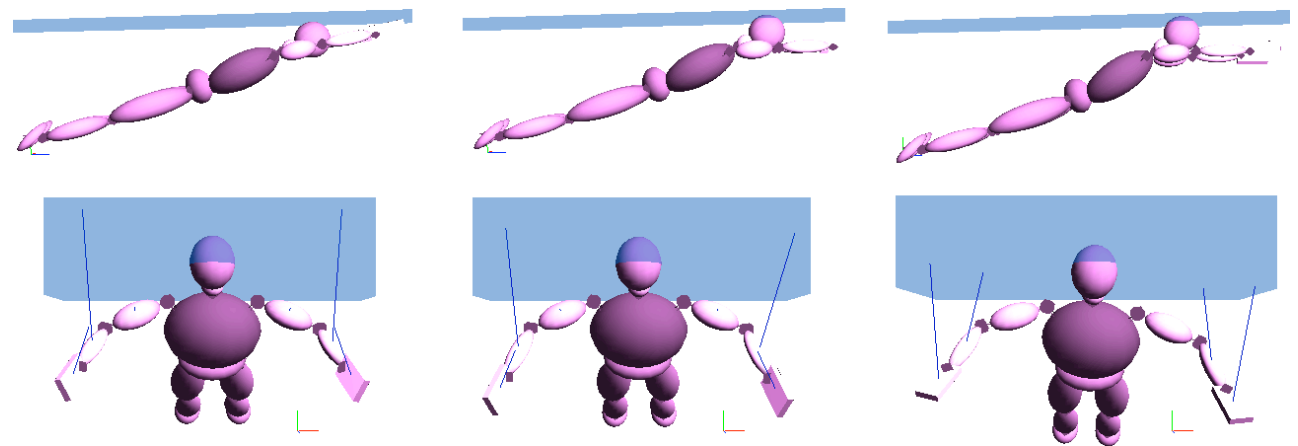

Figure 8: The effect of subsequent layers on the resulting motion. a) Basic stroke layer drives the character's desired pose. $b$ ) A per-cycle perturbation aligns the character's arms with the fluid surface. c) A continuous perturbation reorients the palms to maximize forward thrust on the palm.
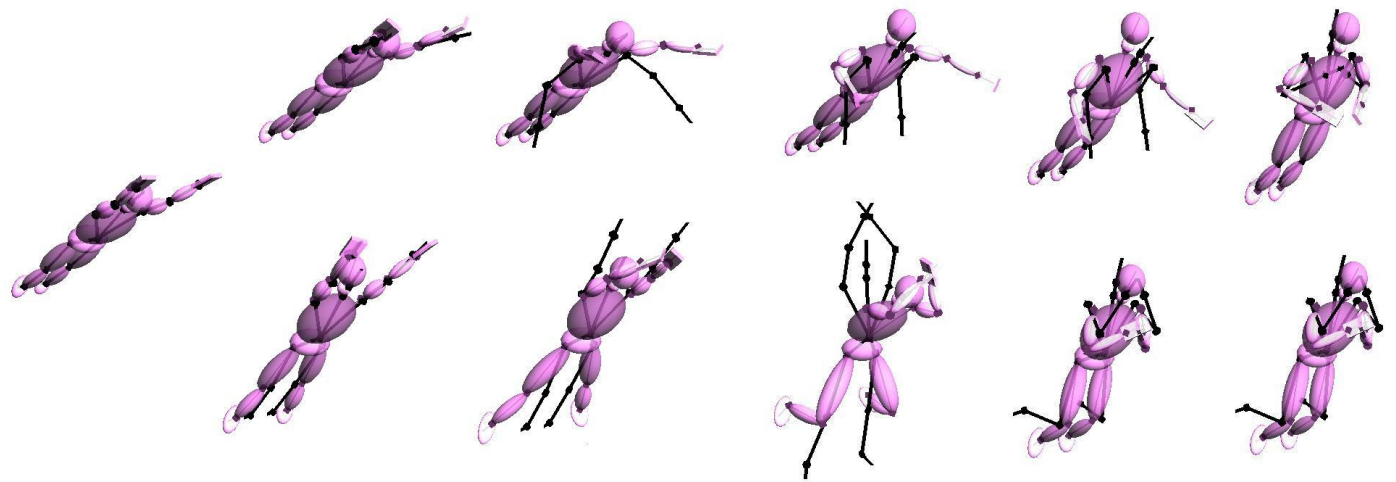

Figure 9: The difference between desired (skeletal line) and the actual poses in simulation. One cycle of the breast stroke is shown (clockwise from left).

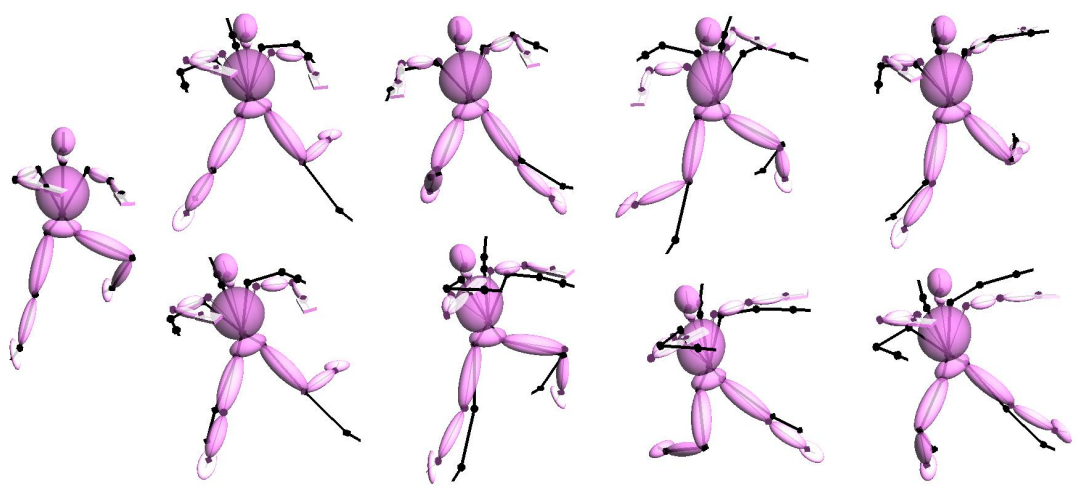

Figure 10: The actual and desired pose of a character turning left while treading water. One cycle is show going clockwise from the left. 
direction. Conversely, the palms should slice through the fluid if less force is desired.

- Arm Reach The arms should reach out further if more force is desired in the current desired direction and the arms should be closer to the torso for less force.

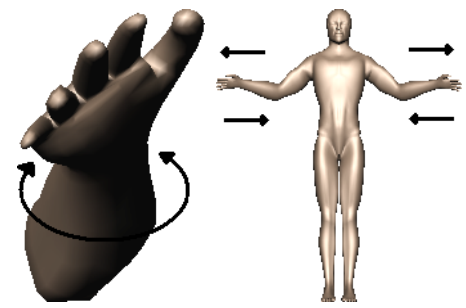

Figure 12: Continuous perturbations: a) palm orientation and $b$ ) arm reach.

Figure 13 demonstrates that applying the palm orientation rule to our basic treading water sequence improves forward propulsion of the swimmer.

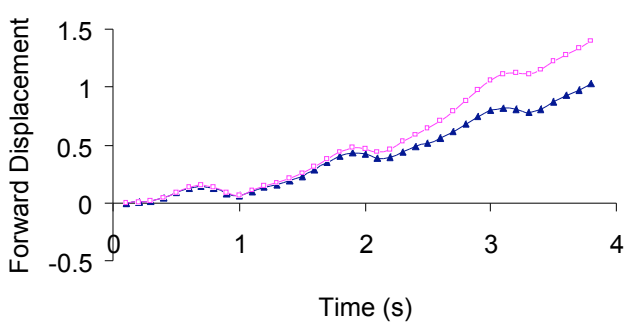

$\rightarrow$ Without Perturbation -- With Palm Perturbation

Figure 13: Effect of palm orientation control on the forward displacement of character's root (pelvis).

\section{Implementation and Results}

The layered algorithm was implemented using the commercial toolkit $S D$ fast. Strokes are defined as keyframed joint angles exported from commercial animation systems. The target is interactively specified by the user with a mouse or read as a trajectory from a file.

To test the capability of our system, we created several different target trajectories for the virtual swimmer to follow. Figure 14 shows the swimmer tracking a figure of eight. Figure 15 shows the effects of subsequent layers on tracking a target with a cross current in the fluid. Note that the basic stroke layer leaves the swimmer entirely at the mercy of environmental forces and unable to follow the target. The percycle perturbation layer enables the character to face into the current every cycle to maintain a horizontal heading. Finally the continuous perturbation layer produces greater thrust, allowing the swimmer to track the target more closely.

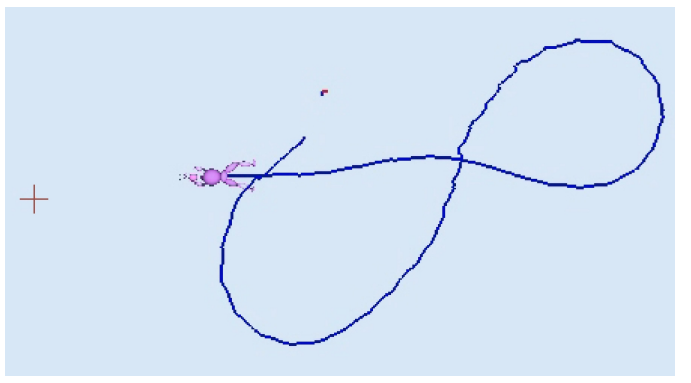

Figure 14: Tracking a figure of eight.

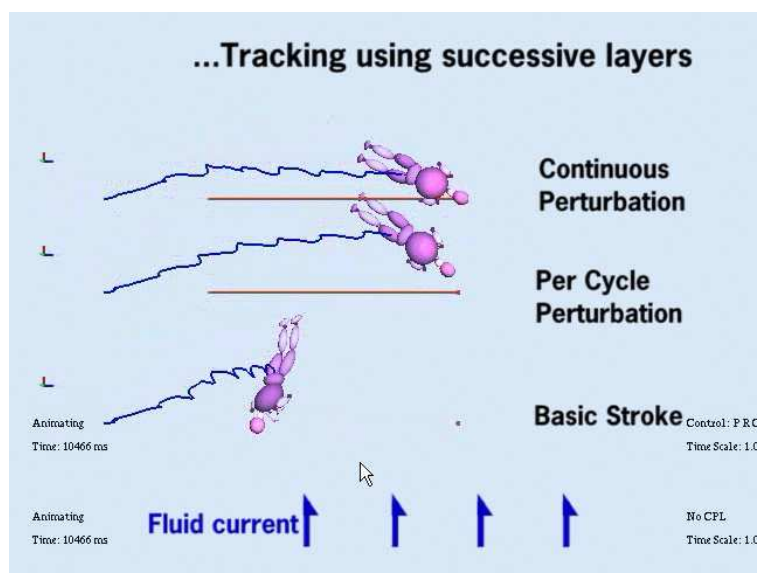

Figure 15: Effect of layers on target tracking in a strong current.

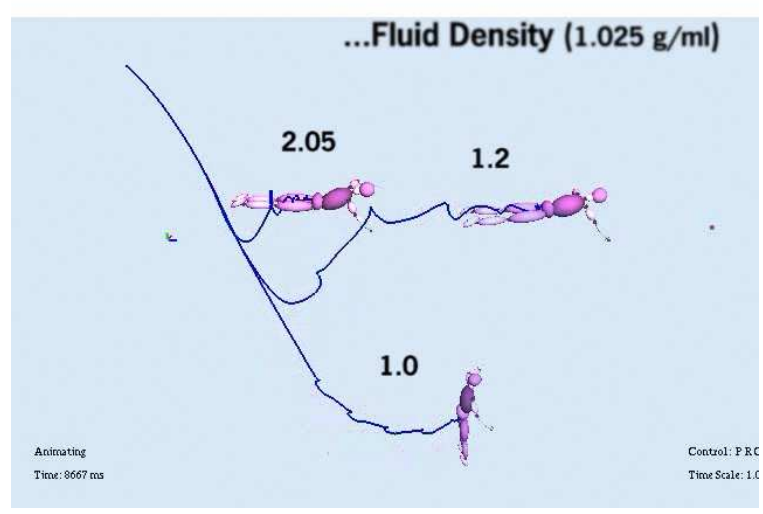

Figure 16: Varying the density of the fluid. 
We also find the approach to be robust to the manipulation of various swimmer and fluid parameters as shown in Figure 16, which shows the swimmer in varying fluid density. Note how the swimmer sinks less with increasing fluid density. The swimmer struggles to recover and follow the horizontally moving target in the least dense fluid due to a lack of sufficient thrust. The swimmer similarly struggles in a very dense fluid on account of a greater drag force.

We implemented three strokes to test the versatility of our approach and visually compared them with video footage of real swimmers. Figure 1 shows the breast stroke, Figure 17 the front crawl and Figure 18 shows the swimmer treading water.

\section{Discussion and Future Work}

The main contribution of this paper is to propose a continuously adaptive layered algorithm for interactively controlling a virtual swimmer in a dynamic environment. The figures in the paper and the accompanying video show the approach to produce visually pleasing results and to be robust to changes in the environment and model parameters.

Currently, our fluid model is unaffected by the motion of the swimmer through it. This is an oversimplification of reality and its biggest drawback is that the thrust generated is lower than that observed in real swimmers. One possible future direction is to incorporate more accurate fluid forces while maintaining interactivity of the simulation using, for example, the panel method [KAT01]. A full CFD simulation with unsteady flow is, however, necessary to truly capture the motion of swimming characters.

There are a large number of unexplored questions in controlling the motion of characters in fluids. At a higher level the selected stroke with which to follow a target is typically specified by the animator. Automating control over stroke selection best capable of following a given target, in a manner similar in spirit to Faloutsos et. al [FvT01] is one future direction. Higher level strategies for the control of multiple swimmers, such as in a water polo game, is a subsequent step. Most importantly, unlike motion on land which has been well studied and can be analyzed using motion capture, movement in water is less explored and its capture using vision techniques is difficult. We hope that this paper will stimulate future work in this area and serve as a tool to coaches and athletes for swimming education and stroke analysis and improvement.

\section{Acknowledgements}

We would like to thank Shahzad Malik and Nigel Morris for their contribution to an early prototype of this research, Jos Stam and Byron McDonald for many fruitful discussions, and Damir Geljo and Tara C., for allowing us to capture reference video of them swimming. Thanks also to the anonymous reviewers.

\section{References}

[BdH*95] Berger M., De Groot G., Hollander A.: Hydrodynamic drag and lift forces on human hand / arm models.In Journal of Biomechanics, vol. 28 (2), Elsevier Science, pp. $125-133.3$

[BR02] BiXlar, B. AND RiEWALD, S.: Analysis of a swimmer's hand and arm in steady flow conditions using computational fluid dynamics. In (Journal of Biomechnics), vol. 35(5), Elsevier Science, pp. 713-717. 3

[CMT04] CARlson, M., Mucha, P. And Turk, G.: Rigid Fluid: Animating the interplay between Rigid Bodies and Fluid. To appear in Proceedings of the 31st Conference on Computer Graphics and Interactive Techniques, ACM Press. 3

[FvT01] Faloutsos, P., VAN DE PANNe, M, AND TERzopolous, D. : Composable controller for physics-based character animation. In Proceedings of the 28th Conference on Computer Graphics and Interactive Techniques, ACM Press, pp. 251-260. 2, 8

[GHD03] GÉnevaux, O., Habibi, A., And DisCHLER, J.: Simulating Fluid-Solid Interaction. In Graphics Interface (GI-03), CIPS, Canadian Human-Computer Commnication Society, A K Peters, pp. 31-38. 3

[GT95] GRZESZCZUK, R., AND TERZOPOLOUS, D.: learning of muscle-acutated locomotionthrough control abstraction. In Proceedings of the 22nd Conference on Computer Graphics and Interactive Techniques, ACM Press, pp. 63-70. 3

[HWB*95] Hodgins, J., Wooten, W., Brogan, D., AND O'BRIEN, J.: Animating Human Athletics. In Proceedings of the 22nd Conference on Computer Graphics and Interactive Techniques, ACM Press, pp. 71-78. 1,2

[HOE65] Hoerner, S.: Fluid-Dynamic Drag, Published by Author, Library on Congress Catalog Card Number: 64-19666 3, 4

[HB75] HoERner, S. ANd Borst, H.: FluidDynamic Lift, Published by Liselotte A. Hoerner. Library on Congress Catalog Card Number: 75-17441 3,4

[KAT01] Katz, J.: Low Speed Aerodynamics, Cambridge University Press. Cambridge, UK. ISBN: 0-5216-6552-3 8

[LvF96] Laszlo J. F., van de Panne M., Fiume 

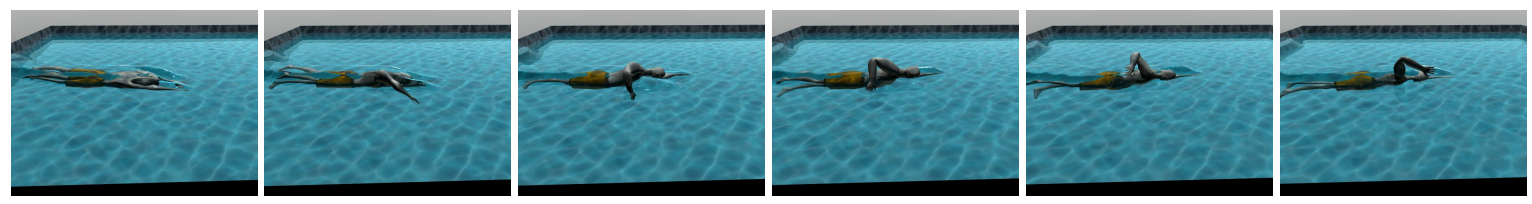

Figure 17: Front crawl.
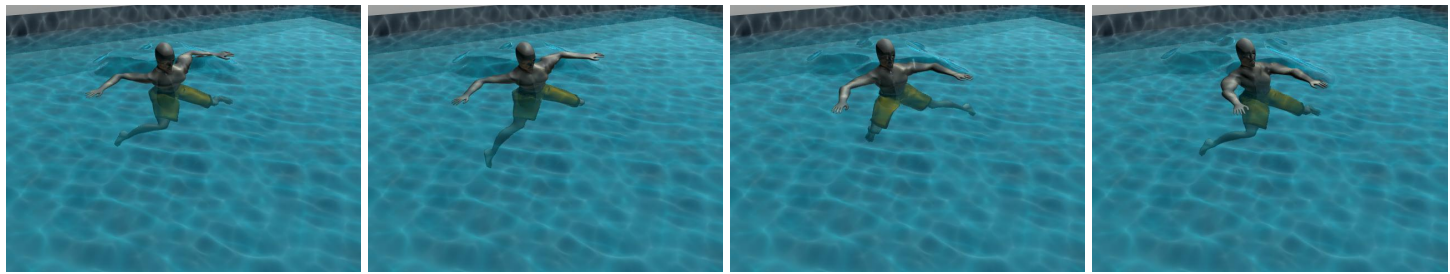

Figure 18: Treading water.

E.: Limit cycle control and its application to the animation of balancing and walking. In SIGGRAPH 96 Conference Proceedings (Aug. 1996), Rushmeier H., (Ed.), Annual Conference Series, ACM SIGGRAPH, Addison Wesley, pp. 155-162. held in New Orleans, Louisiana, 04-09 August 1996. 1, 2

[MAG03] Maglischo, E.: Swimming Fastest, Human Kinetics, ISBN 0-7360-3180-4 2, 3, 5

[MIL88] Miller, G.: The motion dynamics of snakes and worms. In Proceedings of the 15th Conference on Computer Graphics and Interactive Techniques ACM Press, pp. 169173.

[PKH $\left.{ }^{*} 00\right]$ Poyhonen, T., Keskinen, K., Hautala, A., AND MALKIA, E.: Determination of hydrodynamic drag forces and drag coefficients on human leg / foot model during knee exercise. In Clinical Biomechanics, vol. 15, Elsevier Science, pp. 586-593 3

[SH03] SATO, Y. AND HiNO, T.: Estimation of thrust of swimmer's hand using CFD. In Proceedings of the Second International Symposium on Aqua Biomechanism, pp. 71-75. 3

[SIM94] SIMS, K.: Evolving virtual creatures. In Proceedings of the 21st Conference on Computer Graphics and Interactive Techniques, ACM Press, pp. 15-22 1, 3

[STA99] Stams, J.: Stable fluids. In Proceedings of the 26th Conference on Computer Graphics and Interactive Techniques, ACM Press, pp. 121-128.

[TOU02] Toussaint, H.: Biomechanics of propul- sion and drag in front crawl swimming. In Proceedings of the International Symposium on Biomechanics in Sports 3

[TT94] Tu, X., ANd Terzopolous, D.: Artificial fishes: physics, locomotion, perception, behaviour. In Proceedings of the 21 st Conference on Computer Graphics and Interactive Techniques, ACM Press, pp. 43-50. 1, 2

[vF93] VAn DE Panne, M. And Fiume, E.: Sensor-actuator networks. In Proceedings of the 20th Conference on Computer Graphics and Interactive Techniques, ACM Press, pp. 335-342 2

[VOG94] Vogel, S.: Life in moving fluids, University Press, ISBN 0-691-03485-0

[WH91] Wejchert, J., And Haumann, D.: Animation of Aerodynamics. In Proceedings of the 18th Conference on Computer Graphics and Interactive Techniques, ACM Press, pp. 19-22 3, 4

[WH96] Wooten W. L., Hodgins J. K.: Animation of human diving. Computer Graphics Forum 15, 1 (1996), 3-14. ISSN 0167-7055. $1,2,3$

[WP03] WU, J. AND Popović, Z.: Realistic modeling of bird flight animations. In Proceedings of ACM SIGGRAPH 2003 (2003), Hodgins J., Hart J. C., (Eds.), vol. 22(3) of ACM Transactions on Graphics, pp. 888-895. 1, 3,5 